\title{
A Method for Estimating Noise from Full-Scale Distributed Exhaust Nozzles
}

\author{
Kevin W. Kinzie ${ }^{*}$ \\ NASA Langley Research Center, Hampton, VA 23681 \\ David. B. Schein ${ }^{\dagger}$ \\ Northrop Grumman Integrated Systems, El Segundo, CA 90245-2804
}

\begin{abstract}
A method to estimate the full-scale noise suppression from a scale model distributed exhaust nozzle (DEN) is presented. For a conventional scale model exhaust nozzle, Strouhal number scaling using a scale factor related to the nozzle exit area is typically applied that shifts model scale frequency in proportion to the geometric scale factor. However, model scale DEN designs have two inherent length scales. One is associated with the mini-nozzles, whose size do not change in going from model scale to full scale. The other is associated with the overall nozzle exit area which is much smaller than full size. Consequently, lower frequency energy that is generated by the coalesced jet plume should scale to lower frequency, but higher frequency energy generated by individual mini-jets does not shift frequency. In addition, jet-jet acoustic shielding by the array of mini-nozzles is a significant noise reduction effect that may change with DEN model size. A technique has been developed to scale laboratory model spectral data based on the premise that high and low frequency content must be treated differently during the scaling process. The model-scale distributed exhaust spectra are divided into low and high frequency regions that are then adjusted to full scale separately based on different physics-based scaling laws. The regions are then recombined to create an estimate of the full-scale acoustic spectra. These spectra can then be converted to perceived noise levels (PNL). The paper presents the details of this methodology and provides an example of the estimated noise suppression by a distributed exhaust nozzle compared to a round conic nozzle.
\end{abstract}

$\begin{array}{ll}\text { A } & =\text { nozzle area } \\ \text { DEN } & =\text { distributed exhaust nozzle } \\ \text { EPNL } & =\text { effective perceived noise level } \\ \text { NPR } & =\text { nozzle pressure ratio } \\ \text { OASPL } & =\text { overall sound pressure level } \\ \text { PNL } & =\text { perceived noise level } \\ \text { SPL } & =\text { sound pressure level } \\ \mathrm{T} & =\text { Thrust } \\ \theta & =\text { sideline directivity angle relative to nozzle inlet } \\ \phi & =\text { azimuthal angle orientation }\end{array}$

\section{Introduction}

Jet noise continues to be a dominant aircraft noise source that limits operations of current aircraft and hinders the design of future aircraft. While techniques aimed at changing the engine cycle or those implementing mixing enhancement devices are incrementally improving the community noise situation, revolutionary improvements in conventional engine/airframe systems are required to meet NASA's perceived noise reduction goal of $20 \mathrm{~dB}$ in 25

\footnotetext{
* Senior Research Engineer, Aeroacoustics Branch, Hampton, VA, MS 166, AIAA Senior Member

${ }^{\dagger}$ Engineer, Northrop Grumman Integrated Systems, El Segundo, CA, AIAA Member
} 
years. One such concept with potential to make significant progress toward the 25 -year goal is the distributed exhaust nozzle (DEN).

Noise suppression by the DEN results from a favorable shift in the spectral shape of the radiated jet noise. The smaller jets that comprise the distributed exhaust nozzle radiate noise at higher frequencies than larger single or dual flow exhaust nozzles. Atmospheric attenuation increases nearly exponentially with increasing frequency, and spectral noise components contribute less and less to the EPNL noise metric as frequency increases above $4 \mathrm{kHz}$. In fact, noise at frequencies higher than $10 \mathrm{kHz}$ is not even included in the calculation of EPNL. In addition to shifting the noise signature toward more favorable high frequencies, the small jets mix with the ambient air and reduce jet plume speed and temperature to lower levels that result in reduced low frequency noise.

Traditionally, DEN concepts have been studied from the perspective of replacing conventional engine exhaust nozzles with another configuration composed of many small tubes, chutes, or spokes ${ }^{1}$. However, this inevitably leads to high levels of base drag due to the aft facing area required to distribute the exhaust. NASA is conducting research aimed at studying the distributed exhaust concept from an integrated exhaust/airframe system perspective involving integration of the propulsion system into the airframe and distribution of small exhaust nozzles over large portions of the wing surface area ${ }^{2,3}$.

A characteristic of the DEN technology is significant reduction in low frequency noise accompanied by an increase in high frequency components of noise. The crossover point between the low and high frequency features is problematic when it comes to projecting scale model DEN acoustics to full size. Typically, Strouhal number scaling involves applying a factor related to the nozzle exit area that shifts model scale frequency in proportion to the geometric scale factor. If a single factor is used to scale model-scale DEN acoustic spectra, the high frequency cross-over causes DEN data to be much louder than a comparable round nozzle because of the frequencies that dominate the sound field on a PNL basis. However, model scale DEN designs have two length scales. One is associated with the mini-nozzles, which are close to full scale, and the other is associated with the overall nozzle exit area which is much smaller than what it would be full size. Consequently, the lower frequency energy that is generated by the coalesced jet plume should scale to even lower frequency, but the higher frequency energy generated by individual mini-jets will not shift frequency. In addition, jet-jet acoustic shielding by the arrays of nozzles will be a significant noise reduction effect that may increase with DEN model size. In other words, laboratory DEN models should be treated not as scaled models, but as sections of full size nozzles.

\section{Background}

Kinzie et al. ${ }^{3}$ describes small-scale nozzle experiments of two different DEN designs that each showed significant noise reduction compared to a round reference nozzle. However, because of the difficulties just described, no assessment was made of the potential noise reduction in terms of a full-size nozzle. Using the acoustic data from the slanted psuedo-slot (SPS) nozzle, a method will be described here to estimate the noise suppression from such a design on a full-scale basis.

The SPS nozzle was manufactured using a stereolithography process and is shown in Fig. 1. The nozzle has a total exit area of $3.32 \mathrm{in}^{2}$ with individual mini-nozzles having areas of $0.030 \mathrm{in}^{2}$ each. There are seven linear arrays each with 8 mini-nozzles. Additional details of the nozzle design are given in Kinzie et al. ${ }^{3}$. For comparison purposes, a round convergent nozzle with an exit area of $3.14 \mathrm{in}^{2}$ was tested at the same conditions. Several CFD iterations were performed to size the distributed exhaust nozzle such that it had approximately the same mass flow as the round nozzle and therefore the DEN and round nozzle had slightly different exit areas. Figure 2 shows a sketch describing the coordinate system used for this work. An azimuthal angle of $\phi=0^{\circ}$ is the horizontal plane of symmetry and an azimuthal angle of $\phi=90^{\circ}$ is the vertical plan of symmetry. Therefore, for a measurement angle of $\phi=0^{\circ}$, the furthest row of mini-nozzles is shielded from the microphones by the closer rows. For a measurement angle of $\phi=90^{\circ}$, the microphones are directed at all the mini-nozzle rows equally. Polar directivity angles are designated by $\theta$ and are measured from the jet inlet axis with $\theta=180^{\circ}$ being aligned directly on the jet axis in the downstream direction.

The nozzles were tested in NASA Langley's Small Anechoic Jet Facility (SAJF). An eight-element linear microphone array on an approximate 7 foot sideline was used to measure radiated noise. Because of the size of the chamber and of the nozzles, acoustic measurements were only made in the aft quadrant from polar angles of $\theta=90^{\circ}$ to $\theta=155^{\circ}$. Narrowband data up to $100 \mathrm{kHz}$ were acquired with $1 / 4$ " B\&K 4139 microphones and post-processed to standard $1 / 3$ octave bands. The data were then extrapolated to a 12 foot arc centered at the nozzle exit and corrected to standard day reference conditions using the Shields and Bass atmospheric attenuation model ${ }^{4}$. 
A range of NPR conditions were measured ranging from NPR $=1.45$ to NPR $=2.20$. For all pressure conditions, the flow total temperature was held at $120^{\circ} \mathrm{F}$. The flow temperature was limited to relatively cool conditions by the plastic stereolithography models. This paper focuses on the NPR $=1.72$ case an example.

Figure 3 shows measure SPL spectra at two sideline angles for the round reference nozzle and the DEN at NPR $=1.72$. A directivity angle of $\theta=150^{\circ}$ is shown in Fig $3 \mathrm{a}$ and $\theta=90^{\circ}$ is shown in Fig. 3b. At the most downstream angle of $\theta=150^{\circ}$, there is $10-15 \mathrm{~dB}$ reduction in the low frequencies with an $8-10 \mathrm{~dB}$ increase in the high frequencies. The orientation angle of $\phi=0^{\circ}$ is slightly quieter, but the two azimuthal angles have similar spectral shapes. For the sideline directivity angle of $\theta=90^{\circ}$, there is not as much noise suppression from the DEN. In fact, for the $\phi=90^{\circ}$ orientation, the DEN spectrum has a large hump at low frequencies that exceeds the level of the round reference nozzle. This spectral content has been observed in several similar DEN designs ${ }^{3,5}$ and the cause of this excess noise has not yet been determined. Separate experiments by Ahjua indicate that it might be caused by internal nozzle geometry.

CFD calculations of thrust performance show an approximate $11 \%$ performance loss from the DEN relative to the round nozzle. This is a significant decrease and makes the application of the DEN to a conventional aircraft very challenging. However, for a revolutionary aircraft application there is the potential mitigate the nozzle performance penalty through other aircraft design features. Therefore the noise suppression from the DEN is still attractive. An analysis will be shown in Section IV comparing the noise of the DEN and round nozzle on an equal thrust basis.

The difficulty with scaling these laboratory model data to larger sizes is apparent in the spectra of Figs. 3. Using a single scale factor is not appropriate because of the multiple length scales involved and would result in unrealistically large SPL values for the DEN as the high frequency content associated with the mini-nozzles scaled to a lower frequency range. However, because there is at least one order of magnitude difference between the length scale associated with the coalesced jet plume and that associated with the mini-nozzles, it may be possible to identify the energy associated with each of those length scales in the acoustic spectra and scale them differently based upon their characteristic physics. This task requires some knowledge of frequency components associated with the mini-nozzles. Completely blocking off the slots on one side of the nozzle and measuring the noise radiated from both sides of the nozzle provided a first order estimate of acoustic energy generated by the mini-jets close to the nozzle. Noise radiated close to the nozzle surface from the mini-jets will have little acoustic radiation towards microphones on the non-flowing side of the nozzle since most of that energy would be shielded from the microphones. Figure 4 shows the spectra at two sideline directivity angles for NPR $=1.72$ and for two azimuthal orientations $180^{\circ}$ apart. Again, a sideline angle of $\theta=150^{\circ}$ is shown in Fig $3 \mathrm{a}$ and $\theta=90^{\circ}$ is shown in Fig. $3 \mathrm{~b}$. For $\phi=90^{\circ}$, the microphones are directed at the side of the nozzle with the open slots and for $\phi=270^{\circ}$ the microphones are directed at the side of the nozzle with the blocked slots. At low frequency, there is little difference in the noise measured on either side of the nozzle. At higher frequencies there is a dramatic drop in noise for $\phi=270^{\circ}$ as the high frequency sources are shielded from the microphones at this orientation. Therefore, there is evidence that the highest frequencies are generated close to the nozzle by the mini-jets. Since a full scale DEN would contain minijets of approximately the same size as those in the small-scale model, it is reasonable to assume that the spectral content associated with the mini-jets will be similar between the large and small-scale nozzles. Only a change in amplitude will result from the difference in the total number of mini-jets.

\section{Spectral Separation Procedure}

In order to scale a model-scale spectrum to one representative of a full-scale DEN, a process must be established that separates the model spectrum into energy associated with the coalesced jet plume and energy associated with mini-nozzle jet noise. Once the model scale spectrum is separated into these low and high frequency regimes, each part can be scaled separately based on their corresponding physics. The low frequency regime associated with the larger coalesced jet plume should scale with the approximate linear geometric scale factor of the jet. Therefore, as the model scale spectrum is scaled to a larger size, these frequency components will scale down linearly with the geometric scale factor and the amplitude will scale up logarithmically with the area (or the square of the geometric scale factor). However, since the mini-nozzles themselves are already approximately full size, the frequency components associated with them should not be adjusted. Only the amplitude should be scaled based on the difference between the number of mini-nozzles on the model and full-scale nozzle. Once the two frequency regimes have been scaled, they can then be recombined into spectrum that can be used for estimating full-scale OASPL or PNL. 
The first step in the process is to develop high and low frequency filter functions to separate the model scale spectrum into two frequency regimes. This is not a completely objective process, but data such as those shown in Fig. 4 can provide guidance how to divide the energy. Based on the assumption that the mini-jets and the coalesced jet plume both scale as typical jet noise, an approach was developed to separate the DEN acoustic spectrum into multiple spectral components, one representing high frequency mini-nozzle energy and another representing low frequency coalesced jet energy. A third region was also chosen to account for the mid-frequency region between these two. Each component was assumed to have a characteristic "haystack" jet mixing noise spectrum, with the sum of the spectra producing the full measured spectrum from the DEN.

Predicted spectral shapes were taken from the recommended spectra for jet mixing noise developed by Stone and Montegani $^{7}$, which are an element of their semi-empirical model for predicting noise generated by exhausts from circular nozzles. Example spectra are shown in Fig. 5. Note that the spectral shapes vary with directivity angle. Peak frequencies were chosen by overlaying the appropriate spectrum with the low and high frequency "humps" in the measured spectrum, as shown in Fig. 6. While other combinations of the low, middle, and high frequency spectra could have been chosen, the high and mid-frequency spectra were summed to produce a single spectrum denoted "H." The low frequency spectrum was denoted "L." Subtraction of the "H" and "L" spectra from the measured spectrum on a dB basis produced " $H$ " and "L" filter functions, respectively, which are shown in Figure 7. Finally, application of these filters to the measured DEN SPL spectrum produced two separate spectra for use in the scaling analysis. Note that the filter functions do not alter the data at their extreme ends, but just provide physics based method for the spectral roll-off between the two energy regions. The sum of these spectra, shown in Fig. 8, is nearly equivalent to the original measured spectrum. The individual $\mathrm{L}$ and $\mathrm{H}$ filtered spectra can now be adjusted as described earlier.

\section{Spectral Scaling Procedure}

As an example, the model scale data will be scaled to a 2 foot diameter nozzle which is a linear scale factor of 12. Therefore, the L spectra are shifted to a lower frequency by a factor of 12 and the amplitude is increased by 21.6 $\mathrm{dB}$. The amplitude of the $\mathrm{H}$ spectra is modified by $21.6 \mathrm{~dB}$ based upon the overall area increase with no shift in frequency. The $\mathrm{L}$ and $\mathrm{H}$ spectra are added logarithmically and propagated out to a typical sideline distance, producing an estimate of the full-scale DEN spectra. Figure 9 shows the round reference nozzle and DEN spectra at a downstream angle of $150^{\circ}$, scaled to a $2 \mathrm{ft}$ diameter nozzle, and propagated to a sideline distance of $1000 \mathrm{ft}$. The DEN shows a characteristic double hump and demonstrates significant noise reduction over most of the frequency range. The difference in OASPL between the round reference and DEN is shown in Fig. 10. As a point of reference, the lossless spectrum corresponding to that in Fig. 9 is shown in Fig. 11. Note the significant benefit that the DEN provides due to atmospheric attenuation.

It should also be noted that none of the jet-jet interaction benefit demonstrated in Kinzie et al. ${ }^{2}$ has been included up to this point. Kinzie et al. ${ }^{3}$ showed that for high frequencies such as those characterized by the $\mathrm{H}$ spectra, there is significant jet-jet interaction and shielding such that normal logarithmic $\left(10 \log \mathrm{A}_{1} / \mathrm{A}_{2}\right)$ addition of sources does not apply. For most angles, especially those in the most aft direction, the multiplicative factor for the log area ratio was less than the standard value of 10 . Therefore, the full-scale amplitude increase of the $\mathrm{H}$ spectra would be even less than that shown in Fig. 9. By individually examining the shielding data of Kinzie et al. ${ }^{3}$, it is possible to estimate the amount of shielding provided at each angle and to use this estimate in the area scaling rather than the standard value of 10. Table I shows the multiplicative factor determined for each measured angle. The potential impact of the jet-jet interaction and shielding will be further illustrated in the PNL estimates.

Estimates of full-scale spectrum such as that shown in Fig. 9 can be made for all measurement angles. PNL calculations can then be made to determine the suppression potential of the DEN. Figure 12 shows PNL directivity calculations for the round reference nozzle and the DEN scaled to the conditions of Fig. 9. The DEN PNL values are calculated in two ways. The first uses the more conservative value of $10 \log \left(\mathrm{A}_{1} / \mathrm{A}_{2}\right)$ for the area amplitude increase for the $\mathrm{H}$ spectra. The second uses individual multiplicative factors at each sideline directivity angle as shown in Table I. There is an $8-10 \mathrm{~dB}$ reduction by the DEN over the entire measured directivity range for the conservative case. For the case where additional suppression resulting from the jet-jet shielding is taken into account, the suppression is higher, especially in the downstream direction where there is a $20 \mathrm{~dB}$ reduction by the DEN at the most aft angle.

When comparing the acoustic suppression of the DEN to the round reference nozzle, it is important to make the assessment on an equal thrust basis. Knowing that the DEN experienced an $11 \%$ loss in thrust relative to the round nozzle allows the use of Lighthill's ${ }^{8}$ acoustic scaling laws for jet noise to estimate the noise reduction of the round nozzle at the reduced thrust level. However, there are two ways that thrust from the round nozzle can be reduced. 
One is by simply reducing the exit area (or mass flow) of the nozzle while holding the jet velocity and density constant. The other is by reducing the jet velocity while holding the area and density constant. The first method will reduce the noise by $10 \log \left(A_{1} / A_{2}\right)$ and the second method will reduce the noise by $40 \log \left(T_{1} / T_{2}\right)$. Figure 13 shows noise suppression of several configurations as a function of thrust normalized by the thrust of the round nozzle. Based on the suppression shown in Fig. 12, the suppression of the SPS DEN design is shown as $10 \mathrm{~dB}$ with $11 \%$ thrust loss. If the round nozzle were to experience an $11 \%$ thrust loss based on a reduction in area, the associated noise reduction would be $0.5 \mathrm{~dB}$. If the round nozzle thrust was decreased by reducing the jet velocity, the associated noise reduction would $2 \mathrm{~dB}$. Clearly the noise suppression of the DEN is not just a result of reduced thrust. In fact, if the same noise reduction were to be obtained by reducing the velocity of the round nozzle, the thrust would have to be cut by approximately 44\%. This point is also shown on Fig. 13.

\section{Conclusion}

A method was presented to estimate the full-scale noise spectrum of a distributed exhaust nozzle based on model scale test data. The spectral energy was divided into that associated with the coalesced jet plume and that associated with the mini-nozzles. The separate spectra were then scaled based upon appropriate characteristic physics and recombined to generate full-scale spectrum. The full-scale estimates showed a PNL reduction of 8-10 dB over the entire measured directivity range, with significantly more noise reduction in the aft arc when additional noise suppression resulting from jet-jet interaction is taken into account.

\section{Acknowledgements}

Northrop Grumman Corporation, Integrated Systems performed this work under contract NAS1-02046 funded by the Propulsion Airframe Aeroacoustics subtask of NASA's Quiet Aircraft Technology Program. In addition, Mr. Barry Hellman contributed to the shielding measurements while he was a Langley Aerospace Research Summer Scholar. The authors are also grateful to Dr. Krish Ahuja for his insights into distributed exhaust flows.

\section{References}

1 Hubbard, H.H., “Aeroacoustics of Flight Vehicles: Theory and Practice, Vol. 2: Noise Control," NASA Reference Publication 1258, pp. 228-239.

2 Kinzie, K. W., Brown, M. C., Schein, D. B., and Solomon, Jr., W. D., "Measurements and Predictions for a Distributed Exhaust Nozzle," AIAA Paper No. 2001-2236 presented at the 7th AIAA / CEAS Aeroacoustics Conference, Maastricht, The Netherlands, May, 2001.

${ }^{3}$ Kinzie, K. W., Schein, D. B., and Solomon, Jr., W. D., "Experiments and Analyses of Distributed Exhaust Nozzles," AIAA Paper No. 2002-2555 presented at the 8th AIAA / CEAS Aeroacoustics Conference, Breckenridge, CO, June, 2002.

4 Shields, F. D. and Bass, H. E., "A Study of Atmospheric Absorption of High Frequency Noise and Application to Fractional Octave Bands of Noise," NASA Contractor Report 2760, 1976

5 Gaeta, R. J., Ahuja, K. K., Schein, D. B., and Solomon, Jr., W. D., "Large Jet-Noise Reductions Through Distributed Exhausts," AIAA Paper No. 2002-2456 presented at the 8th AIAA / CEAS Aeroacoustics Conference, Breckenridge, Colorado, June, 2002.

6 Ahuja, K.K., private communication.

7 Stone, J. R. and Montegani, F. J., "An Improved Prediction Method for the Noise Generated in Flight by Circular Jets," NASA TM 81470, 1980.

8 Lighthill, M.J., "On Sound Generated Aerodynamically. I. General Theory," Proceedings of the Royal Society of London A, Vol. 211, 1952. 
Table I Experimentally determined area scaling exponent accounting for jet-jet interaction.

\begin{tabular}{|c|c|}
\hline Angle, $\theta$ & Area Scaling Exponent \\
\hline $90^{\circ}$ & 8.3 \\
\hline $100^{\circ}$ & 8.3 \\
\hline $110^{\circ}$ & 8.3 \\
\hline $120^{\circ}$ & 8.3 \\
\hline $130^{\circ}$ & 7.4 \\
\hline $140^{\circ}$ & 4 \\
\hline $150^{\circ}$ & 3.3 \\
\hline
\end{tabular}

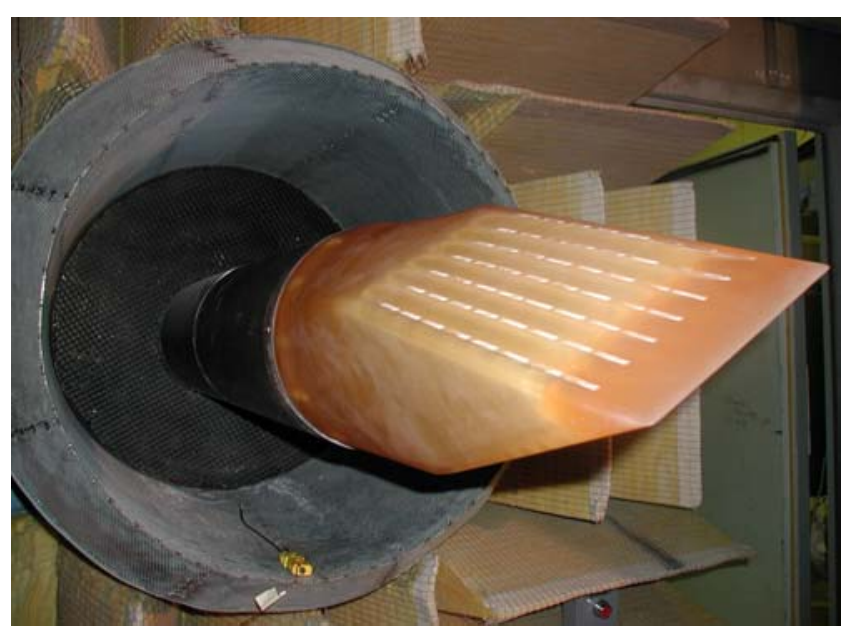

Figure 1 SPS DEN design installed in NASA Small Anechoic Jet Facility.

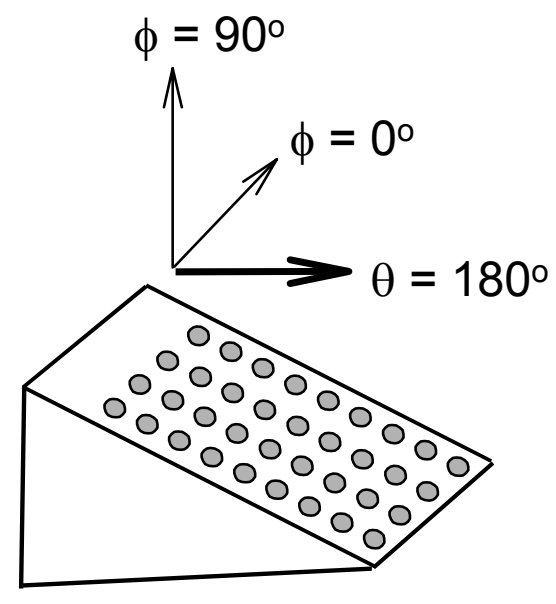

Figure 2 Sketch of nozzle coordinate system and azimuthal orientation planes. 

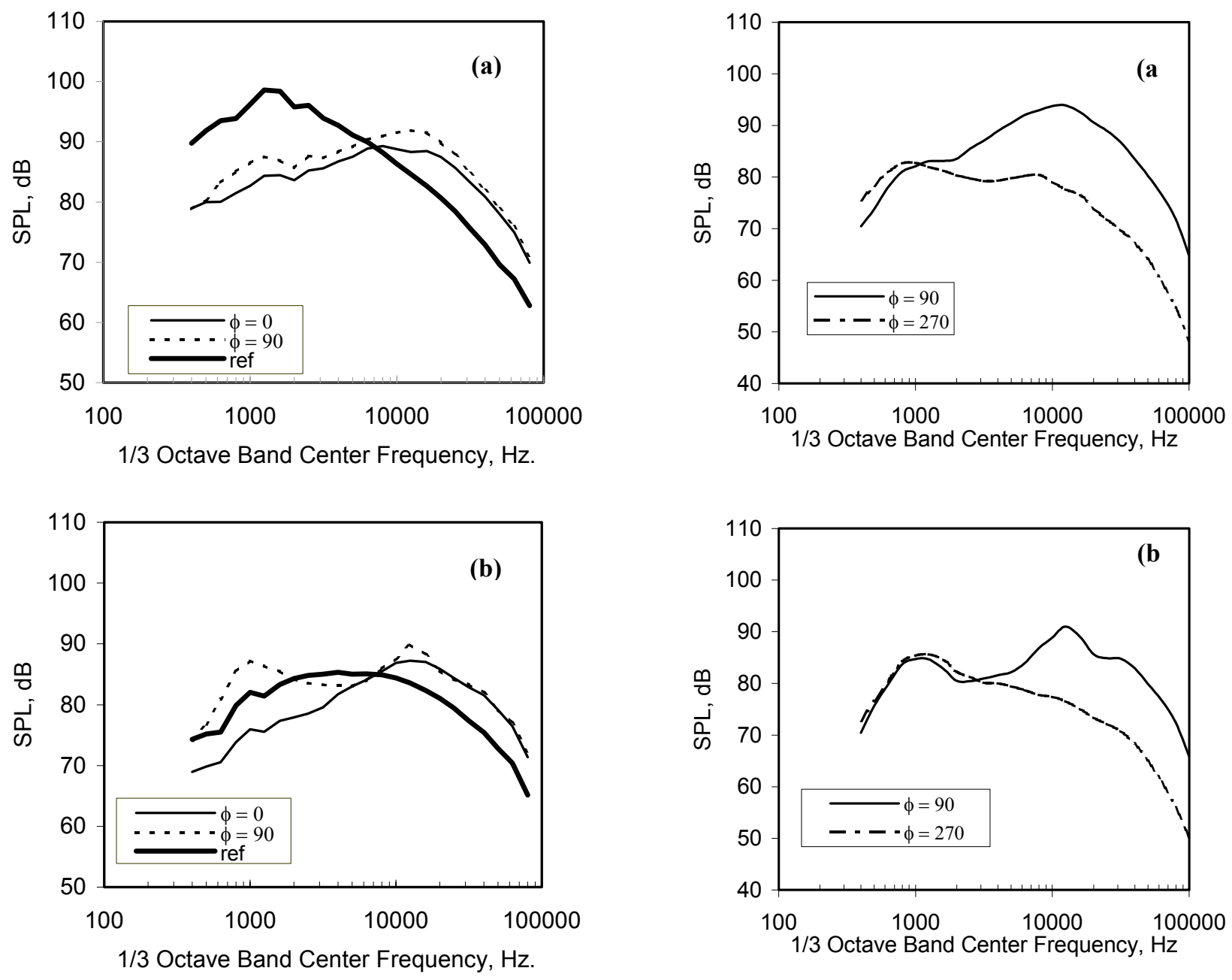

Figure 3 SPL spectra for DEN and round reference nozzle measured at $\mathrm{NPR}=1.72$ a) $\theta=$ $150^{\circ}$; b) $\theta=90^{\circ}$.

Figure 4 SPL spectra for DEN with one side of slots blocked and not flowing a) $\theta=$ $150^{\circ}$ : h) $\theta=90^{\circ}$. 


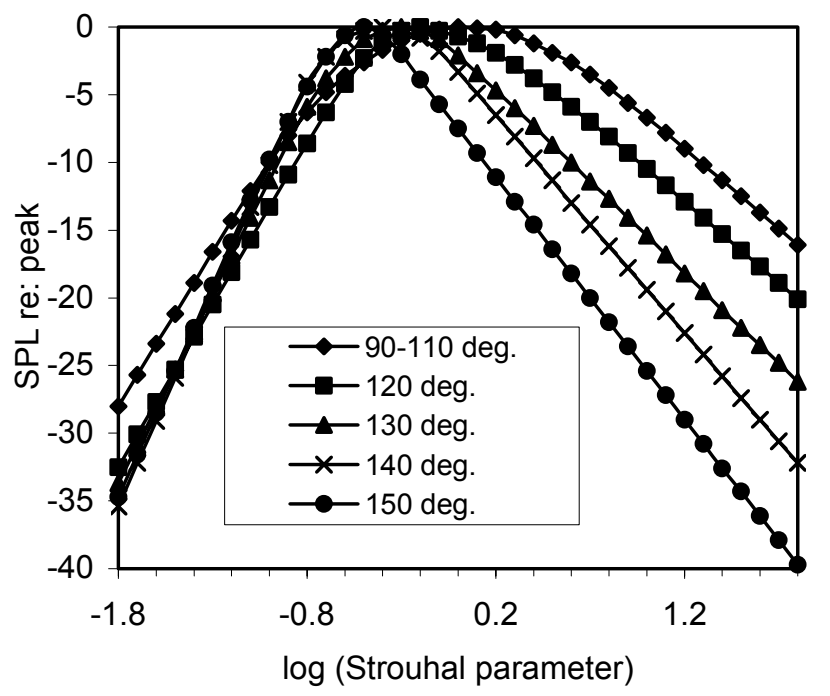

Figure 5 Jet mixing noise spectra from Stone and Montegani ${ }^{5}$.

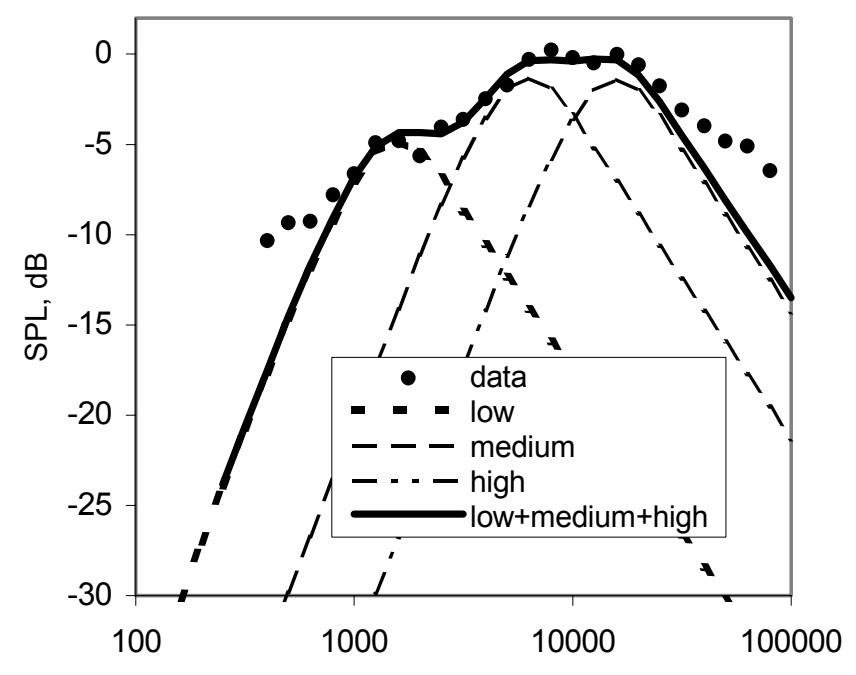

1/3 Octave Band Center Frequency, $\mathrm{Hz}$

Figure 6 Example of spectral source separation process; $\theta=150^{\circ}, \phi=0^{\circ}, \mathrm{NPR}=1.72$.

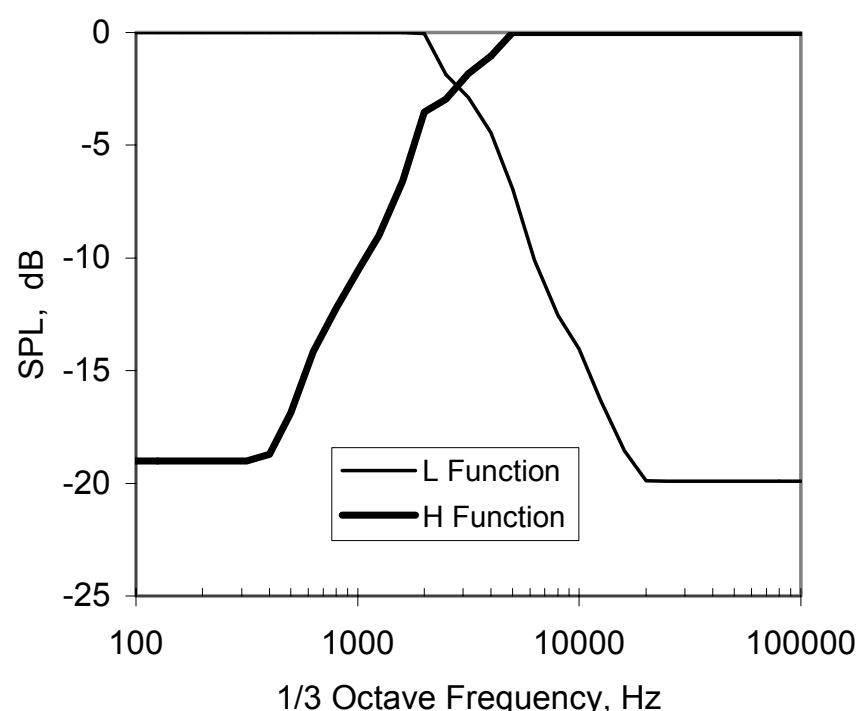

Figure 7 Low $(\mathrm{L})$ and High $(\mathrm{H})$ frequency filter functions applied to data.

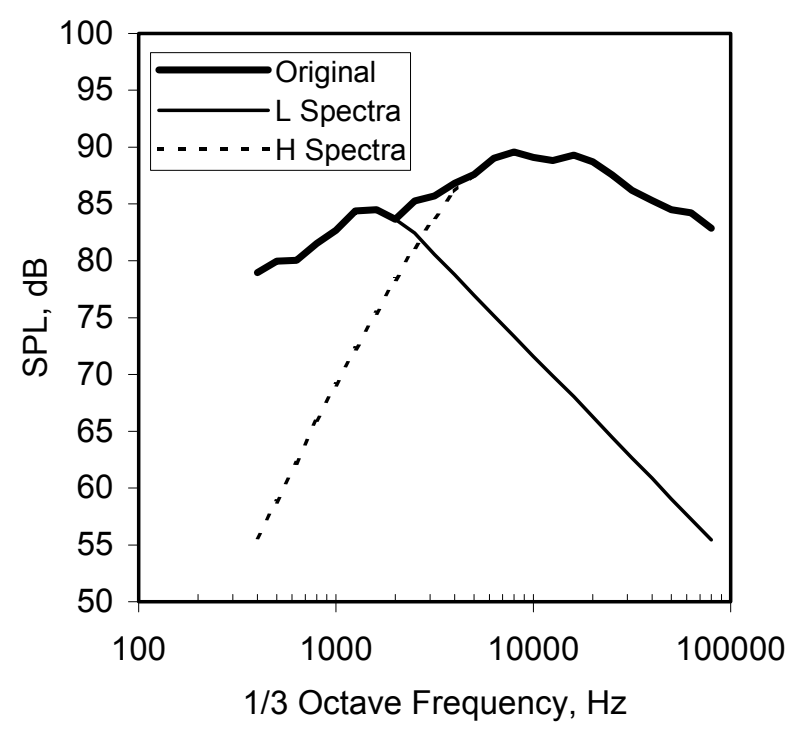

Figure 8 Sample SPL spectra showing Low (L) and High $(H)$ frequency separated source spectra at $\theta=150^{\circ}, \phi=0^{\circ}, N P R=1.72$.

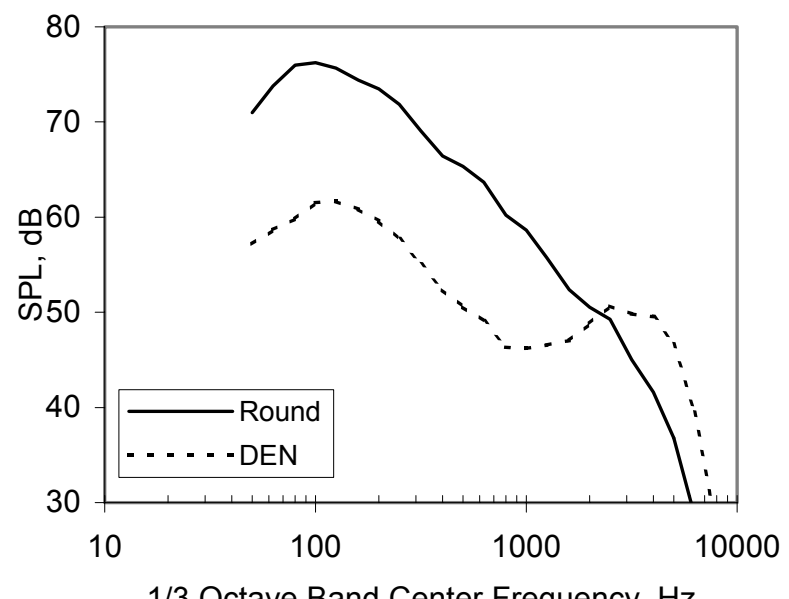

1/3 Octave Band Center Frequency, $\mathrm{Hz}$

Figure 9 Sample spectra of DEN and round nozzle scaled to $2 \mathrm{ft}$ diameter, $1000 \mathrm{ft}$. sideline, with atmospheric attenuation included; $\theta=150^{\circ}, \phi=0^{\circ}, N P R=1.72$

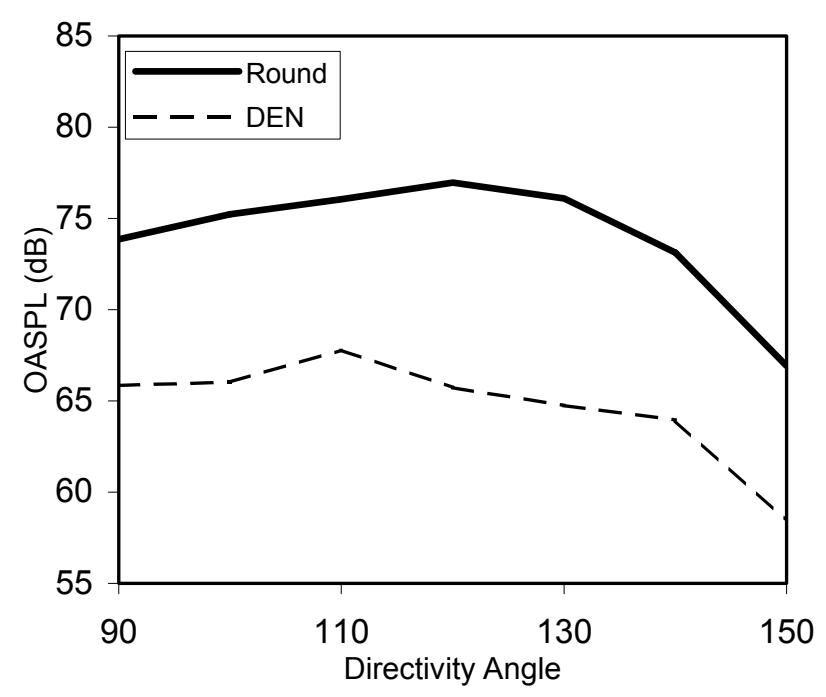

Figure 10 OASPL directivity of round and DEN scaled to $2 \mathrm{ft}$ diameter, $1000 \mathrm{ft}$ sideline, $\phi$ $=0^{\circ}, \mathrm{NPR}=1.72$.

American Institute of Aeronautics and Astronautics 


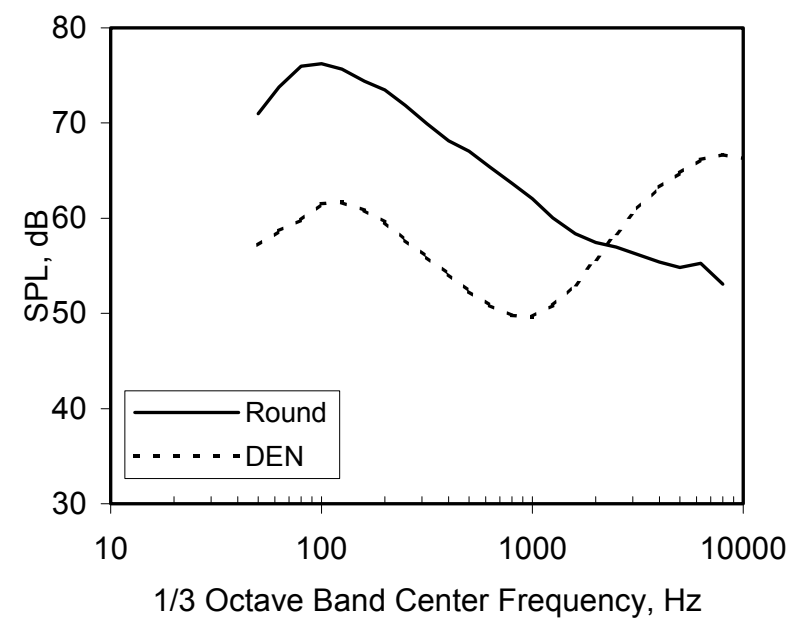

Figure 11 Sample spectra of DEN and round nozzle scaled to $2 \mathrm{ft}$ diameter, $1000 \mathrm{ft}$.

sideline, lossless; $\theta=\mathbf{1 5 0}^{\circ}, \phi=\mathbf{0}^{\circ}, \mathrm{NPR}=1.72$

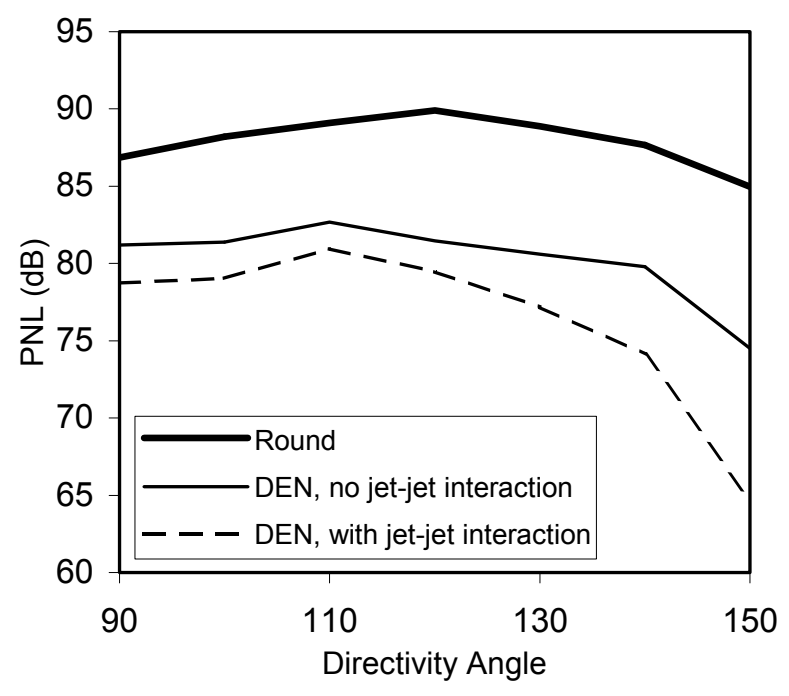

Figure 12 Estimate of Perceived Noise Level for DEN and round nozzle scaled to $2 \mathrm{ft}$ diameter, $1000 \mathrm{ft}$ sideline, $\phi=0^{\circ}, \mathrm{NPR}=1.72$

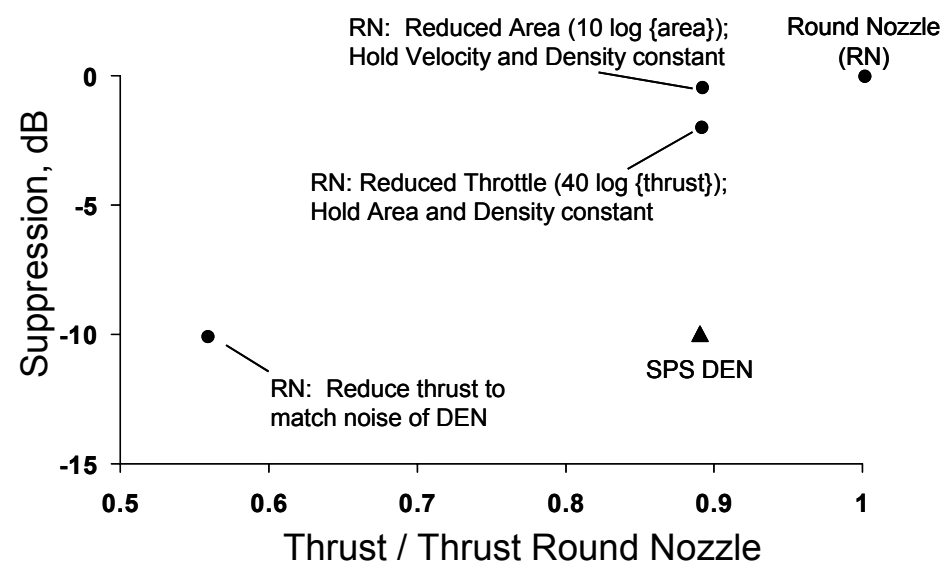

Figure 13 Suppression as a function of thrust loss for the DEN and round nozzle. 